\title{
Energy efficient station for cathodic protection of underground metalic pipelines using pulsed current modes
}

\author{
Alexander Shurayts ${ }^{1}$, Denis Maruhin ${ }^{1, *}$, Hajiahmed Zubailov ${ }^{1}$, and Yury Tomashevskiy ${ }^{2}$ \\ ${ }^{1}$ Gipronigaz JSC, 410012 Saratov, Russia \\ ${ }^{2}$ Yuri Gagarin State Technical University of Saratov, 410054 Saratov, Russia
}

\begin{abstract}
The perspectivity of protection against corrosion by pulsed current in terms of improving the energy efficiency of cathodic protection stations (CPS) are shown. The cathode polarization of the steel structure to the required protective potential values is provided by the station-controlled current parameters, as well as the transition from the direct current mode to the pulse mode and vice versa. A combined load model was implemented, including a traditional electric circuit for replacing a pipeline and a model based on fuzzy rules (FR), which connect the value of protective potential with the state of protective coatings of an underground steel structure, as well as the parameters of exposure to a corrosive environment. The control of the CPS modes using the FR system made it possible to optimize the station's power consumption modes taking into account the variability of external environmental factors affecting the underground pipelines.
\end{abstract}

\section{Introduction}

Reliability and safety in the operation of underground steel structures transporting hydrocarbons can be enhanced by means of an integrated approach to corrosion resistance. The latter includes the use of automated control systems of protective equipment, along with a system for monitoring corrosion rates and forecasting the technical condition of a structure, as well as energy efficient protective methods and technologies. This can be implemented, in particular, by improving active protection [1].

In the works $[2,3]$ the results of laboratory studies of the effect of pulsed current on steel samples are presented. Studies of the amplitude-time characteristics of the increase in polarization when current pulses are applied to the electrochemical cell have shown that the dynamics of the development of the polarization increase (PI) transient is determined mainly by the magnitude of the amplitude current density in the pulse (the smaller amplitude corresponds to the shortest PI transient time) and physicochemical properties «metal - electrolyte» pair. As for the transition process of polarization decay (PD) during the pause, its longer values, achieved by the end of the pulse, correspond to longer PD times. At the same time, the physicochemical properties of the «metal - electrolyte» pair have a significant effect on the PD. It is characteristic that the temporal resolution of the PI transition process is significantly higher than the temporal resolution of the PD transition process and, depending on the parameters of the pulsed current, can exceed it by hundreds or more times.
The results of polarization given in [3] with a pulse mode with a pulse pause parameter of $4 \mathrm{~s}$, a pulse of $0.1 \mathrm{~s}$, demonstrate a level of protection that meets the requirements of regulatory documents while achieving the best physical and mechanical properties (microhardness, tensile strength) of steel properties.

Studies of corrosion resistance methods based on pulse current showed advantages of its use in terms of improving the energy efficiency of cathodic protection stations (CPS) due to transition from direct current (DC) to a pulse mode and vice versa. In these conditions, the station-controlled current parameters provide cathodic polarization of the metallic structure to the required protective potential values $[4,5]$. An implementation of this approach to CPS control results in a marked reduction in power consumption for cathodic protection due to the formation of an appropriate operation mode in relation to actual conditions. It is worth noting that we exclude an overprotection, or inadequate protection of the structures. Such cases are typical of contemporary operational practice. Thus, the extension of the functionality of cathodic protection stations through the use of pulsed modes is an important issue.

\section{Combined model of electrical substitution pattern of pipeline}

Representation of a control object in the process of implementing cathodic protection through its accurate description is a difficult issue. Moreover, it is the basis

\footnotetext{
Corresponding author: marukhin.dn@ gmail.com
} 
of the cybernetic model of the CPS control. When designing cathodic protection stations, which, in addition to the traditional continuous current mode, can operate in a pulsed mode, a load model is necessary taking into account numerous factors characterized by substantial uncertainty, for which fuzzy inference theory was used. The electric circuit for replacing the pipeline was supplemented by a model based on fuzzy rules relating the value of the protective potential to the state of the insulating coatings of the underground structure, as well as the parameters of exposure to a corrosive environment.

As the output current increases, the potential of all points of the structure increases, including near the drain point, the connection point. The increase in the potential is strictly limited not only by normative documents, but also by the physical properties of steel and coating, since as it increases above $-1.2 \mathrm{~V}$ relative to the coppersulphate electrode, hydrogen is facilitated, which accelerates peeling from the metal of the protective coating and penetrates into crystal lattice of metal. This limits the potential and, as a result, the protection area. In order to circumvent the negative effect of the forced operating modes of the cathode installation at the site closest to the anode earthing electrode, to ensure an increase in the potentials at the areas farthest from the cathode station and thereby increase the protection area on extended structures, it would be necessary to redistribute the leakage currents, reducing structure potential in areas where it is unacceptably large, and increasing it in areas where it is unacceptably small. All this is quite difficult to take into account in the analytical model of the load, but it is possible to estimate the protective potential created by the pulsed cathodic protection station, based on fuzzy rules (FR).

The input linguistic variables (LV) were introduced: soil corrosion aggressiveness «Grunt», protective coating condition «Izol», load current «Tok». Accordingly, the output LV will be the magnitude of the protective potential «Potencial». Create Fuzzy Inference Rules. In our case, their number is in the range from 30 to 50 . In this case, it is possible to provide the necessary combination of such properties of the system of FR as redundancy and consistency. Figures 1-3 show the surfaces of the protective potential distribution depending on the pairs of variables («Grunt», «Izol»), («Grunt», «Tok») and («Izol», «Tok») respectively, graphically representing the action 45 synthesized fuzzy rules.

The simulation algorithm using the developed combined model is based on determining the load current value based on the traditional electric circuit equivalent of the pipeline, which later sets the value of the LV «Tok». Other input variables are set by the user based on direct measurements or estimated data calculated based on the normative documentation.

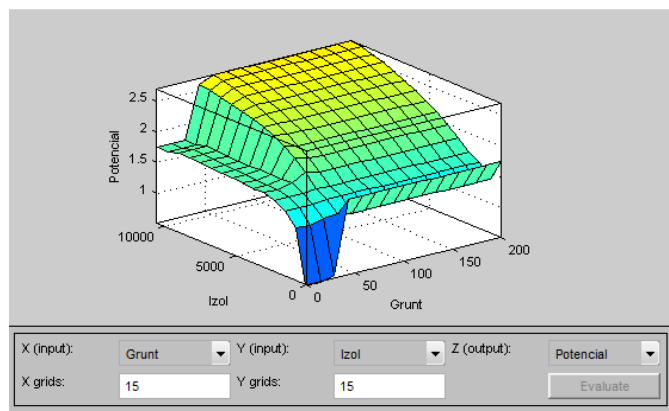

Fig. 1. The dependence of the protective potential of the variables «Grunt» and «Izol».

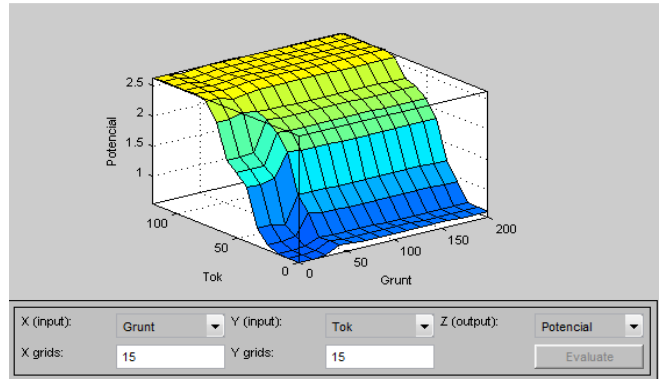

Fig. 2. The dependence of the protective potential of the variables «Grunt» and «Tok».

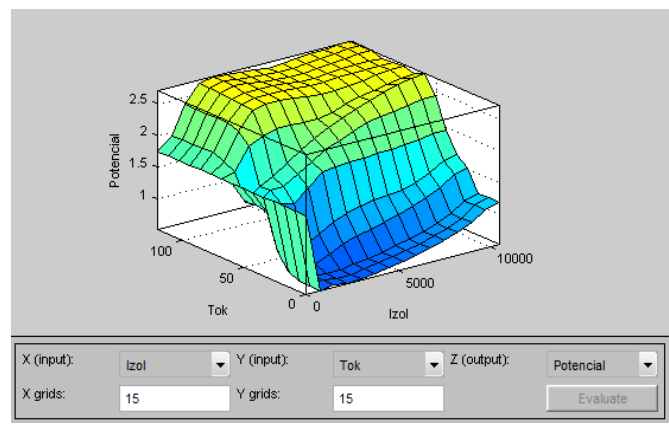

Fig. 3. The dependence of the protective potential of the variables «Tok» and «Izol».

\section{Control of electric power consumption CPS}

Control of CPS modes using the FR allows optimizing the station's power consumption modes taking into account the variability of external environmental factors affecting underground pipelines. As a result of computer simulation was obtained.

1. Translation from continuous to pulsed mode does not significantly change the efficiency. However, with an increase in the equivalent resistance of the pipeline, the efficiency increases. According to the obtained results, an increase in resistance by 3 times reduces the relative loss by about 2.5 times.

2. The magnitude of the relative savings decreases in proportion to the change in the width (duty ratio) of the pulses. 
3. When stabilizing the protective potential, it will determine the optimal point on the dependence of relative savings on the width of the pulses.

The magnitude of the required protective potential is in the narrow range from $-0.85 \mathrm{~V}$ to $-1.1 \mathrm{~V}$. The magnitude of the relative energy savings depends on the change in the width of the pulses, which, in turn, depends on the size of the pipeline, soil condition and insulation resistance. In most practical cases, it is not possible to determine in advance the boundaries and location of the optimal parameter region. Therefore, it is proposed to move to the desired values from the parameters of the pulse mode, which correspond to the maximum energy savings.

Regulation is carried out by a two-circuit control system, the structure of which is shown in figure 4 .

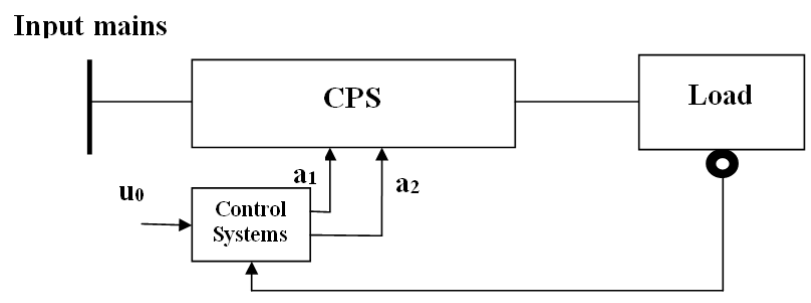

Fig. 4. CPS structure with two control channels.

The first control circuit $\mathbf{a}_{1}$ is connected with the regulation of the amplitude (RA) of the output pulses, the second $\mathbf{a}_{2}$ - with the regulation of the duration (RD) of the output pulses. The first circuit is the master, the second circuit is the slave. In the first circuit, RA is implemented in the range from $U_{\min }$ to $U_{\max }$. When $\mathbf{u}>\mathrm{U}_{\max }$ and $\mathbf{u}<\mathrm{U}_{\min }$, the second $\mathrm{RD}$ circuit is switched on, respectively, increasing or decreasing the width of the output pulses. This scheme allows you to create a constant current in the load, and provide a pulsed mode, while reducing the consumption of electrical energy while fulfilling the requirements to achieve the required polarization of the pipeline.

\section{Simulation results and circuit realization}

Figure 5 shows the Simulink model of a pulse protection system with an inverter link of increased frequency and two control channels: amplitudes and durations of output pulses, complemented by a fuzzy load model.

Figure 6 shows the simulation results of the start-up mode and the change of the Grunt parameter from 30 $\mathrm{ohm} \cdot \mathrm{m}$ to $150 \mathrm{ohm} \cdot \mathrm{m}$ at time $\mathrm{t}=11 \mathrm{~s}$. The upper diagram represents the control signal $\mathbf{a}_{1}$ of the pulse amplitude control channel, the middle image shows the voltage at the load, and the lower one shows the current in the load.

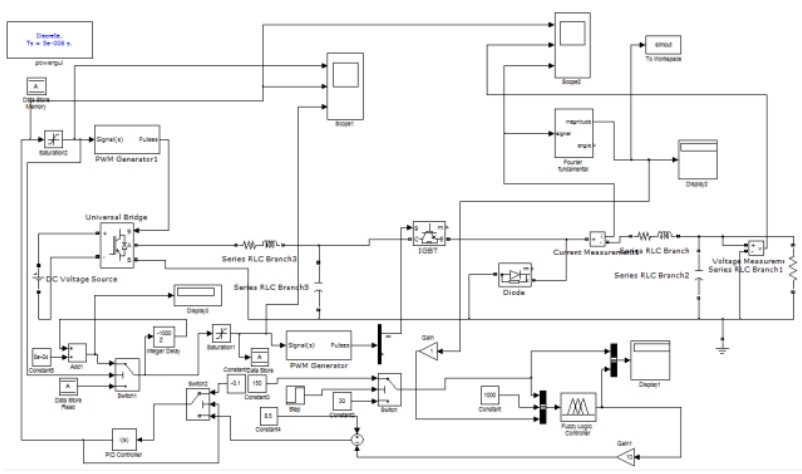

Fig. 5. CPS Simulink model with an inverter link and two control channels: amplitudes and durations of output pulses, including a load fuzzy-model.

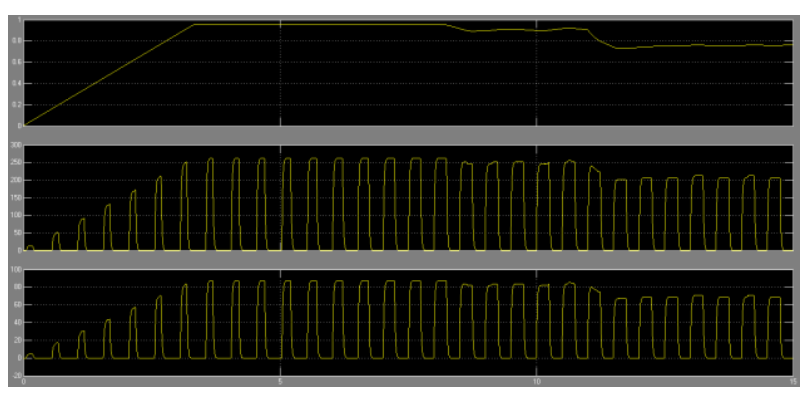

Fig. 6. The diagrams of the start mode and the change of the variable «Grunt» from $30 \mathrm{ohm} \cdot \mathrm{m}$ to $150 \mathrm{ohm} \cdot \mathrm{m}$, the key operates at a frequency of $2 \mathrm{~Hz}$. «Izol»=1000 ohm $\cdot \mathrm{m}^{2}$.

Up to the moment $t=11 \mathrm{~s}$, the CPS provides an exit to the stabilization mode of the protective potential of -0.85 $\mathrm{V}$ according to the proposed algorithm. At first, the control system changes the pulse amplitude, and when the limit value is reached, $U_{\max }$ (at time $t=3.5 \mathrm{~s}$ ) switches to an increase in the pulse duration with a step specified by the Constant 5 unit. At time $\mathrm{t}=8 \mathrm{~s}$, the system returns to the change mode pulse amplitude, as it becomes less than $U_{\max }$. Further, until $\mathrm{t}=11 \mathrm{~s}$, the system maintains the protective potential in this mode. When the value of a «Grunt» is changed, the system stabilizes the protective potential by changing only the amplitude of the pulse at a constant duration.

Based on the simulation results, a basic circuit design was developed for a cathodic protection pulse station, the block diagram of which is presented in Figure 7, where $\mathrm{AC} / \mathrm{DC}$ is a rectifier; $\mathrm{T}$ - power transformer level increased frequency; DC/DC - DC converter; DC/AC inverter, CSRE - copper sulfate reference electrode.

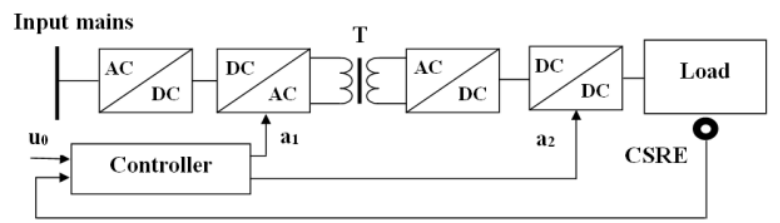

Fig. 7. Block diagram of the basic version of the pulse CPS.

The developed CPS circuitry solution provides DC and pulsed current modes, as well as transitions from one to another, while in a pulsed mode, it implements two- 
channel control of the amplitude and duration of current pulses, respectively.

\section{Conclusion}

1. As the basic parameters of the pulse sequence of cathodic protection stations, in which the corrosion rate decreases to the same polarization of the sample with a direct current, the following are chosen: for the impulse mode - $0.1 \mathrm{~s}$, pause - $4 \mathrm{~s}$. In some cases, due to the transition from the DC mode to the pulse mode and vice versa, the power consumption for cathodic protection is reduced.

2. On the basis of a systematic approach, including the principles of abstraction and decomposition, it became possible to realize a combined load model that takes into account numerous factors characterized by significant uncertainty. In our case, the traditional electric circuit for replacing the pipeline was supplemented by a model based on fuzzy rules (FR), which connect the value of the protective potential with the state of the protective coatings of the underground structure, as well as the parameters of exposure to a corrosive environment.

3. Control of CPS modes using the FR system allows optimizing the station's power consumption modes taking into account the variability of external environmental factors affecting the underground pipelines.

4. A schematic solution of the CPS was developed, which provides DC and pulsed current modes, as well as transitions from one to another, with two-channel control of the amplitude and duration of current pulses, respectively, in a pulsed mode. The use of an automatic installation of the protective potential of a structure by adjusting these parameters makes it possible to increase the reliability of protection and take into account changes in external environmental conditions (soil characteristics, deterioration of passive protection properties).

\section{References}

1. P.R. Roberge, Handbook of Corrosion Engineering, 1130 (1999)

2. D.N. Maruhin, Y.B. Tomashevskiy, Problemy sbora, podgotovki i transporta nefti i nefteproduktov [Problems of Gathering, Treatment and Transportation of Oil and Oil Products], 1, 87-91 (2013) [in Russian]

3. D.N. Maruhin, Y.B. Tomashevskiy, Gazovaya promyshlennost' [GAS Industry of Russia], 7, 56-59 (2014) [in Russian]

4. T.M. Doniguian, Pulsed cathodic protection system and method, Patent US 6224742B1, 10 (2001)

5. D.N. Maruhin, Y.B. Tomashevskiy, Impul'snaya stantsiya katodnoy zashchity podzemnykh sooruzheniy [Pulse cathodic protection station of underground constructions], Patent RF for utility model 122656, 10 (2012) [in Russian] 